\title{
The Generalized Horizontal Linear Complementarity Problem
}

\author{
Bidushi Chakraborty ${ }^{1} \&$ Aniekan A. Ebiefung ${ }^{2}$ \\ ${ }^{1}$ Department of Mathematics, Indian Institute of Technology Kharagpur, India \\ ${ }^{2}$ Department of Mathematics, University of Tennessee Chattanooga, USA \\ Correspondence: Aniekan A. Ebiefung, Department of Mathematics, University of Tennessee, Chattanooga, TN \\ 37403, USA. E-mail: aniekan-ebiefung @utc.edu
}

Received: November 19, 2012 Accepted: December 10, 2012 Online Published: January 8, 2013

doi:10.5539/jmr.v5n1p1 URL: http://dx.doi.org/10.5539/jmr.v5n1p1

\begin{abstract}
Let $M, N \in R^{m \times n}, m \geq n$, be vertical block matrices and $\mathbf{q} \in R^{m}$. We consider the generalized horizontal linear complementarity problem (GHLCP) associated with the pair $\{M, N\}$. Existence and uniqueness of solutions are characterized when the pair $\{M, N\}$ satisfies new generalized column $W$ - and column $W_{0}$-properties.
\end{abstract}

Keywords: linear complementarity problem, generalized horizontal linear complementarity problem, column $W_{0^{-}}$ property, column $W$-property, extended horizontal linear complementarity problem

\section{Introduction}

Given the vertical block matrices $M$ and $N$ of type $\left(m_{1}, m_{2}, \ldots, m_{n}\right)$, the Generalized Horizontal Linear Complementarity Problem (GHLCP) is defined as follows: Find vectors $\mathbf{w}, \mathbf{z} \in R^{n}$ such that

$$
\begin{gathered}
M \mathbf{w}-N \mathbf{z}=\mathbf{q} \\
\mathbf{w} \geq \mathbf{0}, \quad \mathbf{z} \geq \mathbf{0} \\
\mathbf{w}^{T} \mathbf{z}=0
\end{gathered}
$$

where $M, N \in R^{m \times n}, m \geq n, m=\sum_{i=1}^{n} m_{i}$, and $\mathbf{q} \in R^{m}$. We will denote this $\operatorname{problem}$ by $\operatorname{GHLCP}(M, N, \mathbf{q})$.

Problem $\operatorname{GHLCP}(M, N, \mathbf{q})$ is related to the extended linear complementarity problem (LCP) studied by Mangasarian and Pang (1995); the extended horizontal linear complementarity problem (HLCP) studied by Sznajder and Gowda (1995), Schutter and Moor (1997); the generalized LCP studied by Ye (1993); and the generalized horizontal LCP of Xiu and Zhang (2001).

Sznajder and Gowda (1995) extended the concept of $W_{0}$-pair of Wilson (1971). They defined the column $W_{0^{-}}$and column $W$-properties for the set of $(k+1), k \geq 1$, square matrices, and gave some equivalent conditions for the column $W_{0}$ - and column $W$-properties. They also showed that the column $W$-properties are characterized by the unique solvability of the extended HLCP.

In Tütünc $\ddot{u}$ and Todd (1995), properties of a square matrix pair are studied, when the associated matrices have the $P$ - and $P_{0}$-properties. They showed that a large class of matrix pairs including $P_{0}$-pairs have the property of reducibility, and developed an algorithm that can be used to reduce an HLCP to a standard LCP.

Schutter and Moor (1997) considered matrices $A, B \in R^{m \times n}$. They provided an algorithm that can be used to solve the problem and demonstrated that the extended HLCP can be used to solve max-algebraic problems. In their work, Xiu and Zhang (2001) showed that the $\operatorname{GHLCP}(A, B, \mathbf{q})$, where $A, B \in R^{m \times n}, m \geq n$, has a solution when the pair $\{A, B\}$ satisfies the $P_{o^{-}}$and $R_{0}$-properties.

In this paper, we generalize the concepts of column $W_{0}$-property and column $W$-Property to the matrix pair $\{M, N\}$, where $M, N \in R^{m \times n}$ are vertical block matrices of type $\left\{m_{1}, \ldots, m_{n}\right\}$ with $m \geq n$. Using the column $W_{0}$ - and column $W$-Properties, existence and uniqueness of solutions are characterized for the $\operatorname{GLCP}(M, N, \mathbf{q})$. It has been pointed out in Xiu and Zhang (2001) that when $m=n$, the $\operatorname{GHLCP}(M, N, \mathbf{q})$ reduces to the classical horizontal linear complementarity problem $\operatorname{HLCP}(M, N, \mathbf{q})$; and when $M=I_{m \times m}$, the $\operatorname{GHLCP}(M, N, \mathbf{q})$ reduces to the vertical linear complementarity problem studied by Ebiefung (1999).

The HLCP and the extended HLCP have important applications. Eaves and Lemke (1981) showed that any piecewise linear system can be formulated as an HLCP. The extended HLCP has applications in structural mechanics, 
inventory theory, optimization, and statistics as reported in Kaneko (1978) and in Kaneko and Pang (1978). The extended LCP formulated by Schutter and Moor (1997) arose from their work in discrete event systems such as flexible manufacturing, subway traffic network, parallel processing systems, telecommunication systems, and logistic systems.

The rest of the paper is organized as follows: In Section 2, we provide preliminary definitions required for the development of the results. Existence and uniqueness of solutions are provided in Section 3, while concluding remarks are given in Section 4.

\section{Preliminaries}

The following definitions are needed for the rest of this paper.

Definition 1 (See Ebiefung, 1999) Let $M$ be a vertical block matrix of type $\left(m_{1}, m_{2}, \ldots, m_{n}\right)$. An $n \times n$ submatrix of $M$ is called a representative submatrix if its $j$-th row is drawn from the $j$-th block $M^{j}$ of $M$. A vertical block matrix $M$ of type $\left(m_{1}, m_{2}, \ldots, m_{n}\right)$ is a $P_{0}(P)-$ matrix if each representative submatrix is a $P_{0}(P)-$ matrix. A vertical block matrix $M$ of type $\left(m_{1}, m_{2}, \ldots, m_{n}\right)$ has $\prod_{j=1}^{n} m_{j}$ representative submatrices.

Definition 2 (See Sznajder \& Gowda, 1995) Let $\mathcal{M}=\left\{M_{1}, M_{2}\right\}$ be a pair of matrices in $R^{n \times n}$. A matrix $\mathcal{R} \in R^{n \times n}$ is called a column representative of $\mathcal{M}$ if $\mathcal{R}_{. j} \in\left\{\left(M_{1}\right)_{. j},\left(M_{2}\right)_{. j}\right\}, j=1,2, \ldots, n$.

Definition 3 (See Sznajder \& Gowda, 1995) $\mathcal{M}$ has the column $W_{0}$-property if one of the following holds:

(i) The determinants of all column representative matrices of $\mathcal{M}$ are nonnegative and there is at least one such determinant which is positive.

(ii) The determinants of all column representative matrices of $\mathcal{M}$ are nonpositive and there is at least one such determinant which is negative.

Definition 4 (See Sznajder \& Gowda, 1995) $\mathcal{M}$ has the column $W$-property if one of the following holds:

(i) The determinants of all column representative matrices of $\mathcal{M}$ are positive.

(ii) The determinants of all column representative matrices of $\mathcal{M}$ are negative.

Definition 5 (See Ebiefung \& Kostreva, 1993) Let $U$ be a matrix of dimension $n \times m$ defined by:

$$
U=\left(\begin{array}{cccc}
\mathbf{u}^{1} & \mathbf{0} & \ldots & \mathbf{0} \\
\mathbf{0} & \mathbf{u}^{2} & \ldots & \mathbf{0} \\
\ldots & \ldots & \ldots & \ldots \\
\mathbf{0} & \mathbf{0} & \ldots & \mathbf{u}^{\mathbf{n}}
\end{array}\right)
$$

where for each $j(j=1,2, \ldots, n) \mathbf{u}^{\mathbf{j}}$ is a positive row vector of dimension $1 \times m_{j}$.

In the next set of definitions, we consider the $\operatorname{GHLCP}(M, N, \mathbf{q})$ with $M$ and $N$ as vertical block matrices of dimension $m \times n$ and of type $\left(m_{1}, m_{2}, \ldots, m_{n}\right)$, where $(m \geq n)$. That is, $M$ and $N$ are partitioned row-wise into $n$ blocks:

$$
M=\left(\begin{array}{c}
M^{1} \\
M^{2} \\
\cdot \\
\cdot \\
\cdot \\
M^{n}
\end{array}\right) \quad N=\left(\begin{array}{c}
N^{1} \\
N^{2} \\
\cdot \\
\cdot \\
\cdot \\
N^{n}
\end{array}\right)
$$

where the $j$-th blocks $M^{j}$ and $N^{j}$ are of dimension $m_{j} \times n$ and $m=\sum_{j=1}^{n} m_{j}$.

Definition 6 Column matrix: Let $C=\{M, N\}$, where $M$ and $N$ are vertical block matrices of order $R^{m \times n}$. The matrix $\bar{R} \in R^{m \times n}$ defined by

$$
\bar{R}_{. j} \in\left\{M_{. j}, N_{. j}\right\}, \quad j=1,2, \ldots, n
$$

is called a column representative matrix. The matrix $\bar{R}$ is also partitioned to conform with those of $M$ and $N$.

Definition 7 Column $W_{0}$-property: The pair $C=\{M, N\}$ has the column $W_{0}$-property if each column representative matrix is a vertical block $P_{0}$-matrix.

Definition 8 Column $W$-property: The pair $C=\{M, N\}$ has the column $W$-property if all column representative matrices are vertical block $P$-matrices. 
Definition $9 Q$-property: The pair $C=\{M, N\}$ has the $Q$-property if the $\operatorname{GHLCP}(M, N$, q) has a solution for each $\mathbf{q} \in R^{m}$.

Definition 10 Proper matrix: A matrix $B$ defined by $B=\{B . j, j=1,2, \ldots, n\}$, where $B_{. j} \in\left\{M_{. j},-N_{. j}\right\}$, is called a proper matrix of $C=\{M, N\}$ if whenever $\left(-N_{. j}\right)$ is a column of $B, M_{. j}$ is not a column of $B$.

Definition 11 Let $B$ be a proper matrix of $\{M, N\}$. A vector $\mathbf{x}=\left(\mathbf{w}_{\bar{K}}, \mathbf{z}_{K}\right) \geq \mathbf{0}$ is a solution of the $\operatorname{GHLCP}(M, N, \mathbf{q})$ if $B \mathbf{x}=\mathbf{q}$, where $K \subset\{1,2, \ldots, n\}$ and $\bar{K}$ its complement such that $\mathbf{z}_{K} \geq \mathbf{0}, \mathbf{w}_{\bar{K}} \geq \mathbf{0}, \mathbf{z}_{\bar{K}}=\mathbf{0}$ and $\mathbf{w}_{K}=\mathbf{0}$.

Definition 12 A solution $\mathbf{x}=\left(\mathbf{w}_{\bar{K}}, \mathbf{z}_{K}\right)$ of the $\operatorname{GHLCP}(M, N, \mathbf{q})$ is said to be nondegenerate if it has no zero components. We call the $\operatorname{GHLCP}(M, N, \mathbf{q})$ nondegenerate if each solution is nondegenerate.

Definition 13 For each $j(j=1,2, \ldots, n)$, let $\mathbf{e}_{\mathbf{j}}$ be an $m_{j} \times 1$ column vector with each component equal to 1 . The matrix $D$ defined by

$$
D=\left(\begin{array}{cccc}
\mathbf{e}_{1} & 0 & \ldots & 0 \\
0 & \mathbf{e}_{2} & \ldots & 0 \\
\ldots & \ldots & \ldots & \ldots \\
0 & 0 & \ldots & \mathbf{e}_{\mathbf{n}}
\end{array}\right)
$$

is a nonnegative vertical block matrix of type $\left(m_{1}, \ldots, m_{n}\right)$. We assume that $D$ is partitioned to conform to those of $M$ and $N$.

If $M$ is a vertical block matrix, we assume that $M+\alpha D$ has the same partition as $M$ for any $\alpha>0$. We now formulate the perturbed $\operatorname{GHLCP}(M(\alpha), N(\alpha), \mathbf{q})$ :

$$
\begin{gathered}
M(\alpha) \mathbf{w}(\alpha)-N(\alpha) \mathbf{z}(\alpha)=\mathbf{q} \\
\mathbf{w}(\alpha) \geq \mathbf{0}, \quad \mathbf{z}(\alpha) \geq \mathbf{0} \\
\mathbf{w}(\alpha)^{T} \mathbf{z}(\alpha)=0
\end{gathered}
$$

where $M(\alpha)=M+\alpha D$ and $N(\alpha)=N+\alpha D$. Assume that a solution to Equation (2) exists for sufficiently small $\alpha>0$. Also assume that $\mathbf{x}(\alpha)=\left(\mathbf{w}(\alpha)_{\bar{K}}, \quad \mathbf{z}(\alpha)_{K}\right)$ is a solution of Equation (2) and that $B(\alpha)$ is the proper matrix corresponding to $\mathbf{x}(\alpha)$.

The following sequence of solutions of the perturbed problem, Equation(2), will be frequently used in the next section. For each $r \in\{1,2, \ldots\}$, let $\left\{\mathbf{x}\left(\alpha^{r}\right)\right\}=\left\{\left(\mathbf{w}\left(\alpha^{\mathbf{r}}\right)_{\bar{K}}, \mathbf{z}\left(\alpha^{\mathbf{r}}\right)_{K}\right)\right\}$ be the sequence of solutions of $\operatorname{GHLCP}\left(M\left(\alpha^{r}\right), N\left(\alpha^{r}\right)\right.$, $\mathbf{q})$, where $\left\{\alpha^{r}\right\} \rightarrow 0$ as $r \rightarrow \infty$. Moreover, let $\left\{B\left(\alpha^{r}\right)\right\}=\left\{B+\alpha^{r} D\right\}$ be the sequence of proper matrices corresponding to $\left\{\mathbf{x}\left(\alpha^{r}\right)\right\}$ for each $r \in\{1,2, \ldots\}$.

\section{Existence and Uniqueness of Solutions}

The theory of existence and uniqueness of solutions for the GHLCP is presented in this section. Throughout the rest of this paper, the matrix $U$ shall refer to the vertical block matrix defined in Definition $5, B$ the matrix defined in Definition 10, and $D$ the matrix defined in Definition 13.

Theorem 1 If $C=\{M, N\}$ has the column $W_{0}$-property, then $U C=\{U M, U N\}$ has the column $W_{0}$-property.

Proof. Assume that $C=\{M, N\}$ has the column $W_{0}$-property. By definition, each column representative matrix is a vertical block $P_{0}$-matrix. Let $R^{*}$ be a column representative matrix of $U C$. Then $R_{. j}^{*} \in\left\{U M_{. j}, U N_{. j}\right\}, j=1, \ldots, n$. Hence,

$$
R^{*}=U \bar{R}
$$

where $\bar{R}$ is a column representative matrix of $\{M, N\}$.

Since $\bar{R}$ is a vertical block $P_{0}$-matrix, $R^{*}$ is $P_{0}$-matrix of order $n$, as proved by Ebiefung and Kostreva (1993). This is true for each column representative matrix of $U C$. Therefore, $U C=\{U M, U N\}$ has the column $W_{0}$-property. This completes the proof.

Theorem 2 If $C=\{M, N\}$ has the column $W$-property, then $U C=\{U M, U N\}$ has the column $W$-property.

Proof. The proof is similar to the proof of Theorem 1 and so it is omitted.

Theorem 3 Suppose that $C=\{M, N\}$ has the column $W$-property. If the $\operatorname{GHLCP}(M, N, \mathbf{q})$ has a solution, then the solution is unique.

Proof. Suppose that $\{M, N\}$ has the column $W$-property and that the $\operatorname{GLCP}(M, N$, q) has a solution. Assume that $(\mathbf{w}, \mathbf{z})$ is a solution to the $\operatorname{GHLCP}(M, N, \mathbf{q})$. 
Then

$$
\begin{aligned}
M \mathbf{w}-N \mathbf{z} & =\mathbf{q} \\
\mathbf{w} \geq \mathbf{0}, \quad \mathbf{z} & \geq \mathbf{0} \\
\mathbf{w}^{T} \mathbf{z} & =0
\end{aligned}
$$

Multiplying Equation (4) on the left by $U$, we obtain

$$
\begin{aligned}
(U M) \mathbf{w}-(U N) \mathbf{z} & =U \mathbf{q} \\
(U M,-U N)(\mathbf{w}, \quad \mathbf{z})^{T} & =U \mathbf{q}
\end{aligned}
$$

By Equations (5), (6), and (8), (w, z $)^{T}$ solves the $\operatorname{HLCP}(U M, U N, U \mathbf{q})$.

Now suppose that the $\operatorname{GLCP}(M, N, \mathbf{q})$ has two distinct solutions. By the above reasoning, these two solutions are also solutions to the $H L C P(U M, U N, U \mathbf{q})$. However, since $C=\{M, N\}$ has the $W$-property, $U C=\{U M, U N\}$, where $U M$ and $U N$ are square matrices, has the $W$-property by Theorem 2. By Sznajder and Gowda (1995), the $H L C P(U M, U N, U \mathbf{q})$ has a unique solution. This contradicts the assumption that the $\operatorname{GLCP}(M, N, \mathbf{q})$ could have two or more distinct solutions. This completes the proof.

Theorem 4 If $C=\{M, N\}$ has the column $W_{0}$-property, then $C+\alpha D=\{M+\alpha D, N+\alpha D\}$ has the column $W$-property for each sufficiently small $\alpha>0$.

Proof. Assume that $C=\{M, N\}$ has the column $W_{0}$-property. Let $\bar{R}$ be a column representative matrix of $C$. The matrix $\{\bar{R}+\alpha D\}$ is a vertical block $P$-matrix by Ebiefung and Kostreva (1993). This is true for each column representative matrix of $C$. Hence,

$$
C+\alpha D=\{M+\alpha D, N+\alpha D\}
$$

has the column $W$-property for each sufficiently small $\alpha>0$. This completes the proof.

Theorem 5 Suppose that $C=\{M, N\}$ has the column $W_{0}$-property and that the $\operatorname{GHLCP}(M, N, \mathbf{q})$ has a solution with a proper matrix $B$, which is a vertical block $P$-matrix. Then there exists a sequence $\left\{x\left(\alpha^{r}\right)\right\}$ of solutions of the perturbed $\operatorname{GLCP}(M(\alpha), N(\alpha), \mathbf{q})$ which converges to a solution of the $\operatorname{GHLCP}(M, N, \mathbf{q})$.

Proof. Suppose that the $\operatorname{GHLCP}(M, N, \mathbf{q})$ has a solution $(\mathbf{w}, \mathbf{z})$. Then

$$
\begin{gathered}
M \mathbf{w}-N \mathbf{z}=\mathbf{q} \\
\mathbf{w} \geq \mathbf{0}, \mathbf{z} \geq \mathbf{0} \\
\mathbf{w}^{T} \mathbf{z}=0,
\end{gathered}
$$

implies that there exists a vector $\mathbf{x} \in R^{n}$ such that $B \mathbf{x}=\mathbf{q}$, where $\mathbf{x}=\left(\mathbf{w}_{\bar{K}}, \mathbf{z}_{K}\right), \quad \mathbf{q} \in R^{m}, \quad B \in R^{m \times n}$ a proper matrix of the pair $\{M, N\}$. Hence,

$$
\begin{gathered}
\mathbf{B}^{*} \mathbf{x}=\mathbf{q}^{\prime} \\
\mathbf{x}=\left(\mathbf{w}_{\bar{K}}, \quad \mathbf{z}_{K}\right) \\
\mathbf{w}_{\bar{K}} \geq 0, \quad \mathbf{z}_{K} \geq 0 \\
\mathbf{w}_{K}=0, \quad \mathbf{z}_{\bar{K}}=0
\end{gathered}
$$

where $B^{\star}=U B, \mathbf{q}^{\prime}=U \mathbf{q}$. Since $B$ is a vertical block $P$-matrix, $B^{\star}$ is a $P$-matrix by Ebiefung and Kostreva (1993). Thus

$$
\mathbf{x}=\left(B^{\star}\right)^{-1} \mathbf{q}^{\prime} .
$$

Since the $\operatorname{GLCP}(M, N, \mathbf{q})$ has a solution, $\operatorname{GLCP}(M(\alpha), N(\alpha), \mathbf{q})$ has a solution. Let $x\left(\alpha^{r}\right)$ be a sequence of solutions of $\operatorname{GLCP}\left(M\left(\alpha^{r}\right), N\left(\alpha^{r}\right), \mathbf{q}\right)$ and $B\left(\alpha^{r}\right)$ a sequence of the corresponding proper matrices such that

$$
\left(B+\alpha^{r} D\right) \mathbf{x}\left(\alpha^{\mathbf{r}}\right)=\mathbf{q}
$$

for each $r \in\{1,2, \ldots\}$. Then,

$$
U\left(B+\alpha^{r} D\right) \mathbf{x}\left(\alpha^{\mathbf{r}}\right)=\left(B^{\star}+\alpha^{r} D^{\star}\right) \mathbf{x}\left(\alpha^{\mathbf{r}}\right)=\mathbf{q}^{\prime},
$$

where $D^{\star}=U D, B^{\star}=U B, q^{\prime}=U \mathbf{q}$. Thus,

$$
\mathbf{x}\left(\alpha^{\mathbf{r}}\right)=\left(B^{\star}+\alpha^{r} D^{\star}\right)^{-1} \mathbf{q}^{\prime} .
$$


Now taking limits as $r \rightarrow \infty$ and $\alpha^{r} \rightarrow 0$, we obtain

$$
\mathbf{x}^{\prime}=\lim _{\substack{x \rightarrow \infty \\ \alpha^{r} \rightarrow 0}}\left\{\mathbf{x}\left(\alpha^{\mathbf{r}}\right)\right\}=\lim _{\substack{x \rightarrow \infty \\ \alpha^{r} \rightarrow 0}}\left(B^{\star}+\alpha^{r} D^{\star}\right)^{-1} \mathbf{q}^{\prime}=\left(B^{\star}\right)^{-1} \mathbf{q}^{\prime} .
$$

Therefore, $\mathbf{x}^{\prime}=\mathbf{x}$ by (9) and (10). This completes the proof.

Theorem 6 Suppose that $C=\{M, N\}$ has the column $W_{0}$-property and that every proper matrix of $C$ is a vertical block $P_{0}$-matrix. If the $\operatorname{GHLCP}(M, N, \mathbf{q})$ has a nondegenerate solution with a proper matrix $B$, which is also a vertical block P-matrix, then the solution is unique.

Proof. Since the $\operatorname{GHLCP}(M, N, \mathbf{q})$ has a solution, there exists a proper matrix $B$ and a vector $\mathbf{x}=\left(\mathbf{w}_{\bar{K}}, \mathbf{z}_{K}\right)$ such that $B \mathbf{x}=\mathbf{q}$. As a result,

$$
B^{\star} \mathbf{x}=\mathbf{q}^{\prime}
$$

where $B^{\star}=U B, \mathbf{q}^{\prime}=U \mathbf{q}$. Since the $\operatorname{GHLCP}(M, N, \mathbf{q})$ has a nondegenerate solution and $B$ is a vertical block $P$-matrix, $B^{\star}$ is a $P$-matrix, and

$$
\mathbf{x}=\left(B^{\star}\right)^{-1} \mathbf{q}^{\prime}>\mathbf{0}
$$

For $\alpha>0$, let $\mathbf{x}(\alpha)$ be a solution of the perturbed problem $\operatorname{GLCP}(M(\alpha), N(\alpha), \mathbf{q})$ and $B(\alpha)$ the corresponding proper matrix. Then

$$
\begin{gathered}
B(\alpha) \mathbf{x}(\alpha)=\mathbf{q} \\
B^{\star}(\alpha) \mathbf{x}(\alpha)=\mathbf{q}^{\prime}
\end{gathered}
$$

where $B^{\star}(\alpha)=U B+\alpha(U D)=\left(B^{\star}+\alpha D^{\star}\right)$, and $\mathbf{q}^{\prime}=U \mathbf{q}$. Since $B^{\star}$ is a P-matrix, $B^{\star}+\alpha D^{\star}$ is a P-matrix and

$$
\left(B^{\star}+\alpha D^{\star}\right) \mathbf{x}(\alpha)=\mathbf{q}^{\prime} .
$$

for each $\alpha>0$.

Therefore,

$$
\mathbf{x}(\alpha)=\left(B^{\star}+\alpha D^{\star}\right)^{-1} \mathbf{q}^{\prime}>\mathbf{0}
$$

is a nondegenerate solution of the $\operatorname{GHLCP}(M(\alpha), N(\alpha), \mathbf{q})$.

Now suppose that the $\operatorname{GHLCP}(M, N, \mathbf{q})$ has another solution $\overline{\mathbf{x}} \neq \mathbf{x}$ such that $\beta \overline{\mathbf{x}}=\mathbf{q}$, where $\beta$ is a proper matrix of $\{M, N\}$. Since $\beta$ is a vertical block $P_{0}$-matrix, $\beta^{\star}+\alpha D^{\star}$ is a P-matrix for sufficiently small $\alpha>0$. Therefore,

$$
\overline{\mathbf{x}}(\alpha)=\left(\beta^{\star}+\alpha D^{\star}\right)^{-1} \mathbf{q}^{\prime}
$$

is another solution of the $\operatorname{GHLCP}(M(\alpha), N(\alpha), \mathbf{q})$. Thus for a sufficiently small $\alpha>0, \overline{\mathbf{x}}(\alpha)$ and $\mathbf{x}(\alpha)$ are two distinct solutions of the $\operatorname{GHLCP}(M(\alpha), N(\alpha), \mathbf{q})$. This contradicts the fact that the $\operatorname{GHLCP}(M(\alpha, N(\alpha), \mathbf{q})$ should have a unique solution by Theorem 3 .

Theorem 7 Suppose that $C=\{M, N\}$ has the column $W_{0}$-property. If the sequence $\left\{\mathbf{x}\left(\alpha^{r}\right)\right\}$ of solutions of the $\operatorname{GLCP}\left(M\left(\alpha^{r}\right), N\left(\alpha^{r}\right), \mathbf{q}\right)$ is bounded as $\alpha^{r} \longrightarrow 0$, then the $\operatorname{GHLCP}(M, N, \mathbf{q})$ has a solution.

Proof. Since $C=\{M, N\}$ has the column $W_{0}$-property, $(C+\alpha D)$ has the column $W$-property. Assume that

$$
\left\{\mathbf{x}\left(\alpha^{\mathbf{r}}\right)\right\}=\left\{\left(\mathbf{w}\left(\alpha^{\mathbf{r}}\right)_{\bar{K}}, \quad \mathbf{z}\left(\alpha^{\mathbf{r}}\right)_{K}\right)\right\}
$$

is a bounded sequence of solutions of the $\operatorname{GHLCP}\left(M\left(\alpha^{r}\right), N\left(\alpha^{r}\right), \mathbf{q}\right)$ and that $\left\{B\left(\alpha^{r}\right)\right\}$ is the corresponding proper matrix solving the perturbed problem for each $r \in\{1,2, \ldots\}$. Then there exists a subsequence, say, $\left\{\overline{\mathbf{x}}\left(\alpha^{r}\right)\right\}$ of $\left\{\mathbf{x}\left(\alpha^{r}\right)\right\}$ that is convergent. Assume that $\left\{\overline{\mathbf{x}}\left(\alpha^{r}\right)\right\}$ converges to $\mathbf{x}$. Let $\left\{\bar{B}\left(\alpha^{r}\right)\right\}$ be a subsequence of $\left\{B\left(\alpha^{r}\right)\right\}$ corresponding to $\left\{\overline{\mathbf{x}}\left(\alpha^{r}\right)\right\}$ and assume that $\left\{\bar{B}\left(\alpha^{r}\right)\right\}$ converges to $B$. It is easy to see that $B$ is a proper matrix of $\{M, N\}$.

Since

$$
B\left(\alpha^{r}\right) \mathbf{x}\left(\alpha^{r}\right)=\mathbf{q}
$$

for $r=1,2, \ldots$, we have that

$$
\bar{B}\left(\alpha^{r}\right) \overline{\mathbf{x}}\left(\alpha^{r}\right)=\mathbf{q}
$$

This implies that

$$
\lim _{\substack{r \rightarrow \infty \\ \alpha^{r} \rightarrow 0}}\left(\bar{B}+\alpha^{r} D\right) \overline{\mathbf{x}}\left(\alpha^{r}\right)=B \mathbf{x}=\mathbf{q}
$$


Hence, $\mathbf{x}$ is a solution of the $\operatorname{GHLCP}(M, N, \mathbf{q})$. This completes the proof.

\section{Conclusions}

The horizontal linear complementarity problem is extended to the generalized horizontal linear complementarity problem, GHLCP, when associated matrices are vertical block matrices. Existence and uniqueness of solutions of the GHLCP are characterized under generalized column $W_{o^{-}}$and $W$-properties. Under the column $W_{0}$-property, a solution of the GHLCP is obtained by a perturbation technique, which results in a column $W$-property. We showed that if the pair $\{M, N\}$ has the column $W$-property, and the $\operatorname{GHLCP}(M, N, \mathbf{q})$ has a solution, then the solution is unique. As pointed out in the introduction and references, the HLCP has useful applications. It is our hope that the GHLCP will extend and enrich these application areas.

\section{References}

De Schutter, B., \& De Moor, B. (1997). The extented linear complementarity problem and its applications in the max-plus algebra. In Complementarity and Variational Problems:State of the Arts. In M. C. Ferris, \& J. S. Pang (Eds.), SIAM, Philadelphia, Pensylvania.

Eaves, B. C., \& Lemke, C. E. (1981). Equivalence of LCP and PLS. Mathematics of Operation Research, 6, 475-484. http://dx.doi.org/10.1287/moor.6.4.475

Ebiefung, A. A. (1999). The vertical linear complementarity problem associated with $P_{0}$-matrices. Optimization Methods and Software, 10, 747-761. http://dx.doi.org/10.1080/10556789908805739

Ebiefung, A. A., \& Kostreva, M. M. (1993). Generalized $P_{0}$ and Z-matrices. Linear Algebra and Its Applications, 195, 165-179. http://dx.doi.org/10.1016/0024-3795(93)90262-M

Gowda, M. S. (1998). Reducing a monotone horizontal linear complementarity problem to a linear complementarity problem. Applied Mathematics Letter, 8, 97-100. http://dx.doi.org/10.1016/0893-9659(94)00118-V

Kaneko, I. (1978). A linear complementarity problem with n by 2n P-matrix. Mathematical Programming Study, 7, 120-141. http://dx.doi.org/10.1007/BF01395867

Kaneko, I., \& Pang, J.-S. (1978). Some $n$ by $2 n$ linear complementarity problems. Linear Algebra and Its Applications, 34, 297-232.

Mangasarian, O. L., \& Pang, J. S. (1995). The extended linear complementarity problem. SIAM Journal of Matrix Analysis and Applications, 16, 359-368.

Sznajder, R., \& Gowda, M. S. (1995). Generalization of $P_{0^{-}}$and $P$-properties, extended vertical and horizontal linear complementarity problems. Linear Algebra and its Applications, 223/224, 695-715. http://dx.doi.org//10.1016/0024-3795(93)00184-2

Tütüncü, R. H., \& Todd, M. J. (1995). Reducing horizontal linear complementarity problems. Linear Algebra and Its Applications, 223/224, 715-729. http://dx.doi.org/10.1016/0024-3795(94)00147-6

Willson Jr, A. N. (1971). A useful generalization of the $P_{0}$-matrix concept. Numerical Mathematics, 17, 62-70.

Xiu, N., \& Zhang, J. (2001). A smoothing Gauss-Newton method for the generalized horizontal linear complementarity problems. Journal of Computational and Applied Mathematics, 129, 195-208. http://dx.doi.org/10.1016/S0377-0427(00)00550-1

Ye, Y. (1993). A fully polynomial time approximation algorithm for computing a stationary point of the generalized linear complementarity problems. http://dx.doi.org/10.1287/moor.18.2.334

Mathematics of Operations Research, 18, 334-345. 